\title{
Dealing with uncertainty: turbulent parameterizations and grid-spacing effects in numerical modelling of deep moist convective processes
}

\author{
E. Fiori ${ }^{1}$, A. Parodi ${ }^{1}$, and F. Siccardi ${ }^{1,2}$ \\ ${ }^{1}$ CIMA Research Foundation, University Campus of Savona, Savona, Italy \\ ${ }^{2}$ Department of Communication Computer and System, University of Genoa, Genoa, Italy
}

Received: 19 March 2009 - Revised: 18 September 2009 - Accepted: 30 October 2009 - Published: 17 November 2009

\begin{abstract}
Computer power has grown to the point that veryfine-mesh mesoscale modelling is now possible. Going down through scales is clumsily supposed to reduce uncertainty and to improve the predictive ability of the models. This work provides a contribution to understand how the uncertainty in the numerical weather prediction (NWP) of severe weather events is affected by increasing the model grid resolution and by choosing a parameterization which is able to represent turbulent processes at such finer scales.

A deep moist convective scenario, a supercell, in a simplified atmospheric setting is studied by mean of high resolution numerical simulations with COSMO-Model. Different turbulent closures are used and their impacts on the space-time properties of convective fields are discussed. The convectiveresolving solutions adopting Large Eddy Simulation (LES) turbulent closure converge with respect to the overall flow field structure when grid spacing is properly reduced. By comparing the rainfall fields produced by the model on larger scales with those at the convergence scales it's possible to size up the uncertainty introduced by the modelling itself on the predicted ground effects in such simplified scenario.
\end{abstract}

\section{Introduction}

Numerical meteorological forecast at many European and American Meteorological Organizations commonly schedules a drastic reduction of the space scale for the realization of limited-area predictions (Kain et al., 2008; Roberts and Lean, 2008; Xue et al., 2007). Whether this approach can be of real advantage for solving the problems of the uncertainty of the decision-maker, it is doubtful, in particular, for the solution of the problem of nowcasting (Kain et al., 2008; Roberts, 2008; Roberts and Lean, 2008). The extraordinary

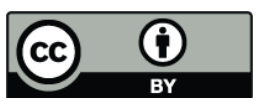

Correspondence to: E. Fiori (elisabetta.fiori@cimafoundation.org) enhancement of the computer power has been promoting a reduction of the modelling horizontal resolution. Obviously such brute-force approach does not guarantee a priori the success.

Two important classes of meteorological numerical approaches are available in the field of simulation of severe weather events with domain size ranging between $3000 \mathrm{~km}$ and $1 \mathrm{~km}$ (Wyngaard, 2004): mesoscale modelling on the larger domains and large-eddy simulation (LES) on the smaller one.

The mesoscale modelling is aimed to produce weather prediction at horizontal resolution of the order of $\mathrm{O}(\sim 10 \mathrm{~km})$ whilst Large Eddy Simulation (LES) is, traditionally, focused on fine scale Planetary Boundary Layer (PBL) convective processes with resolution of the order of $\mathrm{O}(\sim 0.1 \mathrm{~km})$. Figure 1 clarifies their ranges of applicability that did not overlap for many years.

In traditional mesoscale modelling, the energy-containing turbulence scale $l$ is largely exceeded by the typical mesoscale grid-spacing so that little or nothing of turbulence is resolved. Contrary, in LES, the energy and flux-containing turbulence eddies are resolved.

The recent possibility of simulating convective systems with very fine resolution makes the distinction between mesoscale and LES model disappearing (Wyngaard, 2004). In the range of scales between $\Delta_{\text {meso }} \approx 10 \mathrm{~km}$ and $\Delta_{\text {LES }} \approx 0.1 \mathrm{~km}$, a twofold approach is possible to simulate turbulent processes. On the one hand, downscaling the subgrid scale turbulence parameterization used in mesoscale models, on the other hand, upscaling the LES closure. Since neither LES nor mesoscale models were designed to operate in this range, Lilly (1967) named it "terra incognita". Now the interest is to understand which results can be reliably obtained from simulations of deep convective processes realized within "terra incognita" (Wyngaard, 2004), which is a necessary step to assess the amplitude of the contribution to uncertainty stemming up by the modelling itself. 


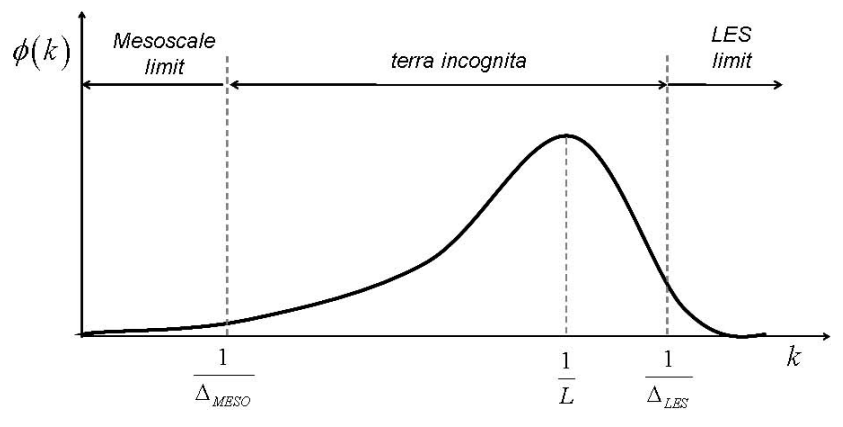

Fig. 1. A schematic view of the turbulence spectrum $\phi(k)$ as a function of the wavenumber magnitude $k$. It peaks at $k \sim 1 / l$, with $l$ the lengthscale of the energetic eddies. $\Delta$ is the model grid-spacing. In the mesoscale limit (left side), $l \ll \Delta_{\text {meso }}$ while in the LES limit (right side), $l \gg \Delta_{\text {LES }}$ (adapted from Wyngaard, 2004).

In the last decades, a significant number of studies have been devoted to test the sensitivity of the numerical simulations in modelling atmospheric phenomena using very fine horizontal resolutions. On the basis of previous non hydrostatic cloud models simulations employing grid resolutions of $2 \mathrm{~km}$ or less (Rotunno et al., 1988; Weisman and Klemp, 1986; Skamarock et al., 1994) Weisman et al. (1997) investigated the effects of horizontal grid size between 1 and $12 \mathrm{~km}$ on the representation of convective process. Changes in terms of timescale of the convective evolution, rainfall rates, intensity and size of the simulated convective system were found. Grabowski et al. (1998) performed two- and three-dimensional simulations of cloud systems showing that model resolution affects the upper-tropospheric cloud cover and consequently the upper-tropospheric temperature tendency due to radiative flux divergence. Petch et al. (2002), in order to obtain a satisfactory representation of the transport of moisture from the sub-cloud layer into the free troposphere, in both shallow and deep convection cases, found that horizontal grid-spacing should be less than one quarter of the sub-cloud layer depth. Adlerman and Droegemeier (2002) approached a simulation problem very similar to the one described in next section. They found that the simulated supercell exhibiting repeated cycling when solved at $\Delta \sim 1 \mathrm{~km}$ evolved into a nearly steady-state unicellular storm at coarser grid-spacing of $2 \mathrm{~km}$. Bryan et al. (2003) studied squall lines from a turbulence perspective using grid-spacings between $1 \mathrm{~km}$ and $0.125 \mathrm{~km}$. The results revealed that simulations with high resolution produce changes in terms of precipitation amount, system phase speed, cloud depth, static stability values, size of thunderstorm cells, and organizational mode of convective overturning as compared to coarser-resolution simulations. The statistical properties of the simulated squall lines were demonstrated still not converging between the $0.25-\mathrm{km}$ and $0.125-\mathrm{km}$ resolution runs.
Along the same lines, this work aims to contribute to the understanding of the uncertainty in numerical weather prediction (NWP) of severe weather events due to different combinations of turbulent parameterizations and fine gridspacings.

A deep moist convective process in a simplified atmospheric scenario, a supercell, is solved by mean of high resolution numerical simulations with COSMO-Model at different grid-spacings with two turbulent closure parameterizations.

Since the middle of 1900's, many studies and numerical experiments have been conducted to evaluate the structure, longevity (Klemp and Wilhelmson, 1978b) and propagation (Rotunno and Klemp, 1985; hereafter indicated as RK85) of severe convective storms. Some of these studies identified the main responsible factors for the development of right (left) moving storms. Weisman and Klemp (1982, 1984, 1986; hereafter indicated as WK82, WK84, WK86) identified buoyancy and vertical wind shear as the two parameters responsible for storm structure and evolution and WK84 also demonstrated that a steady supercellular growth is due to high curved hodographs. WK86 highlights how the shape of the vertical wind profile can strongly influence the form that the convection motion might take. In a strong shear condition for clockwise-curved wind shear profiles, the authors showed a quasi-steady cyclonically rotating updraft development from a short-lived cell due to the growth of a pressure forcing on the right flank of the original updraft.

In this work, we demonstrate that the turbulent closure parameterizations and the resolution do influence the predictive ability (Paola et al., 2006) of COSMO-Model in modelling the evolution of a supercell. We demonstrate that a horizontal grid-spacing exists under which the convective-resolving solutions does converge with respect to the overall flow field structure.

By comparing the rainfall fields produced by the model run at coarse resolution with the ones at the convergence grid-spacing, the uncertainty produced by subgrid smallscale processes on the resolved ground effects is shown. The amount of uncertainty due to the parameterization of subgrid turbulent processes at a certain resolution is reduced when a finer resolution is adopted so that a significant part of those processes are not longer parameterized.

The paper is organized as follows. Section 2 provides a brief overview on the limited area model and its setting on which the deep moist convective system is simulated. Section 3 describes the results in terms of kinematics of the phenomena whilst Sect. 4 compares the rainfall fields produced by graupel and rain sedimentations with different setting for turbulent closure and grid-spacing. Section 5 discusses the results and possible conclusions. 


\section{The convective scenario}

\subsection{Initial conditions and numerical method}

A supercell convection scenario has been chosen for this study. Two are the main reasons for simulating supercell phenomena: first of all, considering the analysis of deep convective scenario in the literature (WK82, WK84, WK86, and RK85), a supercell seems to be a good test-bed for numerical experiments. The well-known dynamical evolution of the supercell makes changes in results of simulations, due to changes in both turbulent closures and grid-spacing, relatively easy to recognise. Secondly, research findings have suggested that, supercell can be occasionally prolific precipitation producers (Moller et al., 1990, 1994; Doswell et al., 1996). On the basis of these considerations an analysis of this convection scenario might offer an improvement on the understanding of the uncertainty affecting the prediction of severe weather events which may be the cause of flash floods in basin where the hydrological response timescales is very short.

The supercell is developed by initializing the model according to WK82 and WK84's numerical experiments. The model is run over a three-dimensional domain that is $150 \mathrm{~km}$ in both cross-line (x) and along-line (y) directions and $20 \mathrm{~km}$ in the vertical. The distance among the 60 vertical levels is stretched gradually from about $80 \mathrm{~m}$ near the bottom boundary to about $500 \mathrm{~m}$ near the top. The adopted horizontal grid-spacings are $1 \mathrm{~km}, 0.5 \mathrm{~km}, 0.25 \mathrm{~km}$ and $0.2 \mathrm{~km}$. A thermal bubble is superimposed on a horizontal homogeneous field. The horizontal radius is $10 \mathrm{~km}$ and the vertical one $1.4 \mathrm{~km}$ with a temperature in excess of $2^{\circ} \mathrm{C}$ at the center that gradually vanishes towards its edge. The bubble is placed at $(25 \mathrm{~km}, 25 \mathrm{~km})$ in the $x-y$ domain to avoid influence of the boundary on its development in the first time steps of integration. For the whole set of the experiments, the initial condition is represented by a directionally varying wind shear that determines supercell motion. The shear vector turns through $180^{\circ}$ over the lowest $5 \mathrm{~km}$ of the atmosphere and becomes constant and unidirectional over $5 \mathrm{~km}$ (Fig. 2). The curvature of the clockwise-curved hodograph is strong enough to favour a right-moving supercell. The thermodynamics conditions make the CAPE value of the order of $1600-1700 \mathrm{~J} \mathrm{~kg}^{-1}$, which means a moderately unstable environment for lifted parcels (Fig. 3).

The sources of uncertainty due to errors in defining initial condition are out of the scope of the present analysis, which only aims to assess the uncertainty due to model setting. Obviously, the representation of the radiation uncertainty in the interaction with soil and vegetation in the surface layer is disregarded (Bougeault, 1997). Modelling of turbulence and its effect on cloud microphysics are the only sources of uncertainty.

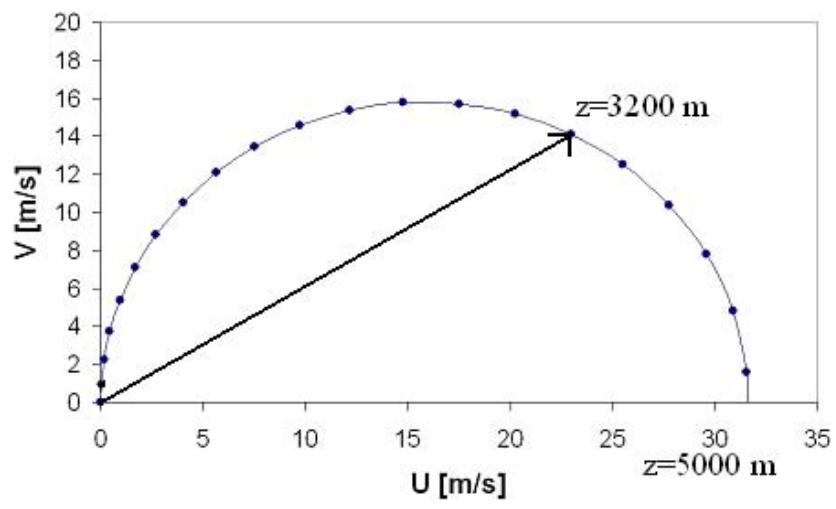

Fig. 2. Wind hodograph. Dots denote the increasing levels, from left to right, in the first $5 \mathrm{~km}$. Wind is kept constant above $5 \mathrm{~km}$.

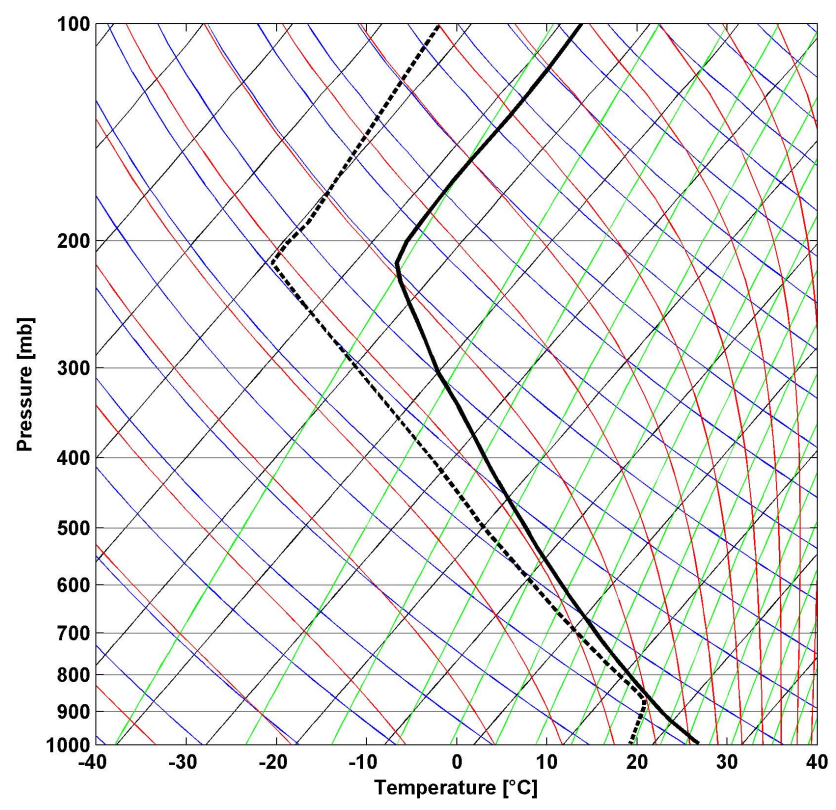

Fig. 3. Skew-T diagram depicting dew point temperature (heavy dashed line) and temperature (heavy solid line) profiles used in numerical simulations. The green lines depict the saturation mixing ratio. Blue ones, the dry adiabats. Red ones, the moist adiabats. Tilted black ones, the isotherms.

The work is performed by using the limited area model COSMO-Model version 3.2. The COSMO-Model is a nonhydrostatic and fully compressible numerical weather prediction model created in 1998 by the Deutsche Wetterdienst (DWD, German National Weather Service). It is developed ever since, in the context of the COSMO consortium, and used for operational purposes by several national and regional meteorological services in Europe. The physics of the model is based on a wide range of turbulent, surface and microphysical schemes. For a more comprehensive description of the model, the reader is referred to Steppeler et al. (2003). 
The experiments are realized neglecting orography and Coriolis forces. Davies (1976) and free-slip conditions are applied to the lateral and ground boundaries, respectively. Rayleigh layer characterizes the top- $5 \mathrm{~km}$ of the atmosphere. The mode-splitting time integration method is a 3rd-order Runge-Kutta scheme and the model is integrated up to $3 \mathrm{~h}$. The adopted time steps are 6, 3, 1.5, and $1 \mathrm{~s}$ for runs from $1 \mathrm{~km}$ to $0.2 \mathrm{~km}$. The high horizontal resolution ranging from $\Delta=1 \mathrm{~km}$ to $\Delta=0.2 \mathrm{~km}$, the lower range of convection permitting and the range of convection resolving, according to Kain et al. (2008), allows us to switch off the convection parameterisation.

Two different turbulent closures available in COSMOModel are used for the four adopted horizontal grid-spacings. Thus, eight numerical experiments were performed.

\subsection{The turbulent schemes}

The approach adopted by COSMO-Model is the traditional one-and-a-half-order approximation, i.e. a turbulent scheme based on the treatment of the prognostic turbulent kinetic energy (TKE).

The two COSMO's closures used herein are called 1D and LES. They differ from each other not only in the way the eddy viscosity coefficient $K$ is calculated but also for the form of TKE equations adopted.

The 1D closure makes use of the so-called boundary-layer approximation by imposing a horizontal homogeneity of the variables. Horizontal fluxes are neglected. The only active turbulent diffusion coefficients for the momentum $\left(K^{M}\right)$ and heat $\left(K^{H}\right)$ equations are those in the vertical direction $\left(K_{V}^{M, H}\right)^{1}$. They are calculated as function of the turbulent length scale $(\lambda)$ (Blackadar, 1962), the prognostic turbulent velocity scale $(q)$ and two stability functions $\left(\sigma_{M}, \sigma_{H}\right)$, depending on the Richardson number (Buzzi, 2008). Thus,

$K_{V}^{M}=q \lambda \sigma_{M}$

$K_{V}^{H}=q \lambda \sigma_{H}$

The horizontal diffusion is simply controlled by the 4 th-order computation mixing coefficient.

The boundary-layer approximation influences the way the shear production term (hereinafter $\mathrm{SP}_{M}$ ) in the TKEequation is calculated. Thus, in 1D-closure the $\mathrm{SP}_{M}$ is composed by the vertical shear of the horizontal wind $(U, V)$ only, e.g.

$\mathrm{SP}_{M}=K_{V}^{M}\left[\left(\frac{\partial U}{\partial z}\right)^{2}+\left(\frac{\partial V}{\partial z}\right)^{2}\right]$

The LES closure uses a three-dimensional subgrid-scale model instead of the boundary-layer approximation. Both

\footnotetext{
${ }^{1} K_{V}^{M, H}$ means vertical turbulent diffusion coefficient for momentum (superscript $M$ ) and heat (superscript $H$ ).
}

vertical and horizontal turbulent coefficients are active. In particular, the vertical coefficients $\left(K_{V}^{M, H}\right)$ are evaluated in according to the equations

$K_{V}^{M}=e^{1 / 2} \lambda \phi_{M}$

$K_{V}^{H}=e^{1 / 2} \lambda \phi_{H}$

where $e$ is the TKE, $\lambda$ is the turbulent length scale, $\phi_{M}$ and $\phi_{H}$ are stability-dependent parameter, depending on the Richardson number (Doms et al., 2005).

Instead, the horizontal $K_{H}^{M, H}$ are simply proportional to the vertical $K_{V}^{M, H}$, through a factor depending on the anisotropy of the mesh, e.g.

$K_{H}^{M, H} \approx r \sqrt{(2)} \frac{\Delta}{\Delta z} K_{V}^{M, H}$

where $r$ is the mean radius of the earth's surface, $\Delta z$ is the elevation step and $\Delta$ is the grid spacing.

In LES closure, the $\mathrm{SP}_{M}$-term of TKE-equation is a function of the vertical $\left(S_{V}\right)$ and horizontal $\left(S_{H}\right)$ components of the deformation squared strain tensor ${ }^{2} S_{i j}^{2}$, which means a three-dimensional behaviour of the production of TKE, i.e.

$\mathrm{SP}_{M}=K_{H}^{M} S_{H}^{2}+K_{V}^{M} S_{V}^{2}$.

\subsection{The microphysical parameterization}

The mathematical description of the overall evolution of a cloud is made in COSMO-Model by the use of a bulk watercontinuity scheme in which only a few categories of water are used and the shape and size distributions of the particles are assumed, while the basic microphysical processes are parameterized.

The dependent variables are the total mass fractions $q^{x}$ of hydrometeors, which are distinguished between nonprecipitating (cloud water $x=c$ and cloud ice $x=i$ ) and precipitating (rainwater $x=r$, snow $x=s$ and graupel $x=g$ ) particles. The precipitating ones are defined by a MarshallPalmer distribution (Marshall and Palmer, 1948):

$f_{x}=N_{0}^{x} e^{-\lambda_{x} D} \quad x=r, s, g$

where $N_{0}^{x}$ is the intercept value we did set to the constant value typical of rainwater, snow and light graupel. The slope parameter $\lambda_{x}$ is determined by the predicted mass fraction $q^{x}$ by inverting the integral

$q^{x}=\frac{N_{0}^{x}}{\rho} \int_{0}^{\infty} m(D) e^{-\lambda_{x} D} d D \quad x=r, s, g$.

\footnotetext{
${ }^{2}$ Deformation strain tensor by components: $S_{i j}=\left(\partial<U_{i}>/ \partial x_{j}+\partial<U_{j}>/ \partial x_{i}\right) / 2$
} 
The corresponding budget equations solved for the many forms of water substances read

$$
\begin{aligned}
& \frac{\partial q^{x}}{\partial t}=A_{q^{x}}+S^{x} \quad x=c, i \\
& \frac{\partial q^{x}}{\partial t}=A_{q^{x}}+\frac{1}{\rho} \frac{\partial P_{x}}{\partial z}+S^{x} \quad x=s, r, g
\end{aligned}
$$

where $A_{q^{x}}$ represents the tendencies for each $x$-type particle, due to processes in which water content behaves as a passive scalar. $P_{x}=\rho q^{x} v_{T}^{x}$, being $v_{T}^{x}$ the mean terminal velocities of the $x$-type particles, represent the sedimentation fluxes of the $x$-type precipitating water category.

The $S^{x}$-terms describe the sources and sinks for each $x$ type water category due to various microphysical transfers between the hydrometeors. Many bulk parameterizations are available in COSMO-Model, from warm rain to threecategory ice scheme.

\section{Results}

\subsection{Storm path}

The supercell numerically modelled in this work is a right moving updraft as in WK86 due to the kinematic initial conditions depicted in Fig. 2. The vertical wind profile is a moderate curved-hodograph which induces one major rightmoving updraft on the right flank of the system and many short-lived thunderstorm systems on the left flank of the gust front. Although all the 8 simulations show this general dynamics, differences in kinematics properties are evident in terms of growth of the convective tower and splitting processes. The initiation of convection is slower at coarse resolution: a longer time is necessary to obtain a complete cell splitting process. Such differences in kinematics are made evident by the inspection of the velocity patterns at different elevations where the effects of both resolutions and turbulent closure are clarified. For sake of conciseness, however, the attention has been paid to the major right-moving cell as the precipitation activity is mainly related to it (Moller et al., 1990, 1994; Doswell et al., 1996). Firstly, the entire path of each cell was captured by tracking its core as the pixel which contains the maximum vertical velocity at the elevation of $5 \mathrm{~km}$. The paths obtained in the first two hours with 1D- and LES-closure are plotted in Figs. 4 and 5, respectively, by a sequence of dots $90 \mathrm{~s}$ apart. Each sequence in both figures corresponds to a given resolution, from $\Delta=1 \mathrm{~km}$ to $\Delta=0.2 \mathrm{~km}$.

The curvature of the path increases as the resolution decreases. Such divergence is very large for 1D-closure (Fig. 4) whilst for LES closure (Fig. 5) the paths corresponding to $\Delta=0.5,0.25$, and $0.2 \mathrm{~km}$ collapse each on the other.

Comparing Figs. 4 and 5 one major result is evident: by using a LES-closure the paths of the right-moving cell does not change when the resolution is increased below $\Delta=0.5 \mathrm{~km}$ : in some a global sense the LES-based simulations do converge at fine resolution. This consideration is not true when $1 \mathrm{D}$ closure is adopted because the convergence of the paths is not yet reached at $\Delta=0.2 \mathrm{~km}$. Now, let us assume the $0.2 \mathrm{~km}$ LES simulation as our reference solution and the corresponding predicted rainfall pattern as our ground truth. If the reader accepts this assumption, the coarse simulation, with $1 \mathrm{D}$ turbulence closure, can be considered as representative of a current state-of-the-art prediction when an operational deterministic cloud-permitting model is used. Comparing the extreme paths, i.e. $1 \mathrm{~km}-1 \mathrm{D}$ run (brown path) and $0.2 \mathrm{~km}$ LES run (blue path), as in Fig. 6, a difference of $\sim 20 \mathrm{~km}$ between the two right-moving cell propagations can be estimated. The case under study is a highly idealized one. If we would like to interpret it in terms of uncertainty for a realtime forecast of a rainfall event, this difference suggests that the choice of the turbulent parameterization scheme and of the grid resolution remarkably impact the forecast: it would imply to warn an area that probably would not receive that precipitation. The uncertainty in numerical modelling using the $1 \mathrm{~km}-1 \mathrm{D}$ setting in fact is also evident by comparing corresponding horizontal cross-sections of the vertical velocity. The pictures on the left-upper and right-lower corners of the Fig. 6 are also reported in Fig. 7: they represent a snapshot of the vertical velocity $\mathrm{W}$ field after $1.5 \mathrm{~h}$ from the run initialization at an elevation of $1.5 \mathrm{~km}$ above ground level (AGL). It is evident that the two settings generate different organization in the convective field. In the $1 \mathrm{~km}-1 \mathrm{D}$-case (panel A in Figs. 6 and 7) a typical supercell-pattern is obtained since a major cell is formed on the right side of the gust front while some well defined convective tower is on the left side. In the LES at $\Delta x=0.2 \mathrm{~km}$ (panel B in Figs. 6 and 7 ) the presence of more intense features on the left flank of the gust front, which is a direct consequence of the tree-dimensional characteristic of the LES-closure, suggests a dynamics of the convective process like a mesoscale convective system.

The uncertainty related with the position of the main rightconvective tower is also evident looking at the panels A and $\mathrm{B}$ : the $0.2 \mathrm{~km}$-LES major cell is at the bottom of the region while the $1 \mathrm{~km}-1 \mathrm{D}$ cell is simply shifted towards right with respect to the centre of picture. In the same time step the maximum updraft velocity in the major cell of the $1 \mathrm{~km}-1 \mathrm{D}$ run is recorded at $(92 \mathrm{~km}, 50 \mathrm{~km})$ (panel A in Fig. 7) while it is at $(86 \mathrm{~km}, 39 \mathrm{~km})$ for the $0.2 \mathrm{~km}-L E S$ run (panel $B$ in Fig. 7).

\subsection{Rainfall depth pattern}

Supercells modelled with $1 \mathrm{~km}-1 \mathrm{D}$ and $0.2 \mathrm{~km}$-LES closures have shown divergent paths of the right-moving cells and different updraft patterns. As already said the case under study is a highly idealized one. However, if we would like to interpret it from a hydrological point of view, a difference of a several kilometres in the main precipitating cell position could be quite irrelevant for large catchments but not 


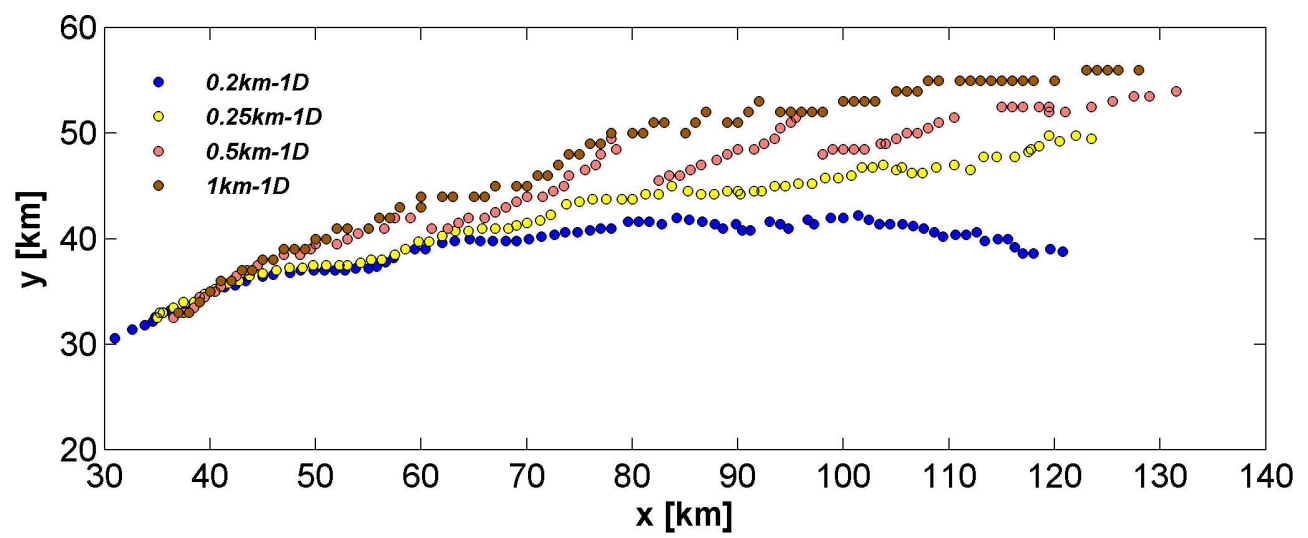

Fig. 4. Path of the core of the right-moving cell modelled using $1 \mathrm{D}$-closure. Brown dots are for the run with $\Delta x=1 \mathrm{~km}$, pink dots are for $\Delta x=0.5 \mathrm{~km}$, yellow dots for $\Delta x=0.25 \mathrm{~km}$ and blue ones for $\Delta x=0.2 \mathrm{~km}$.

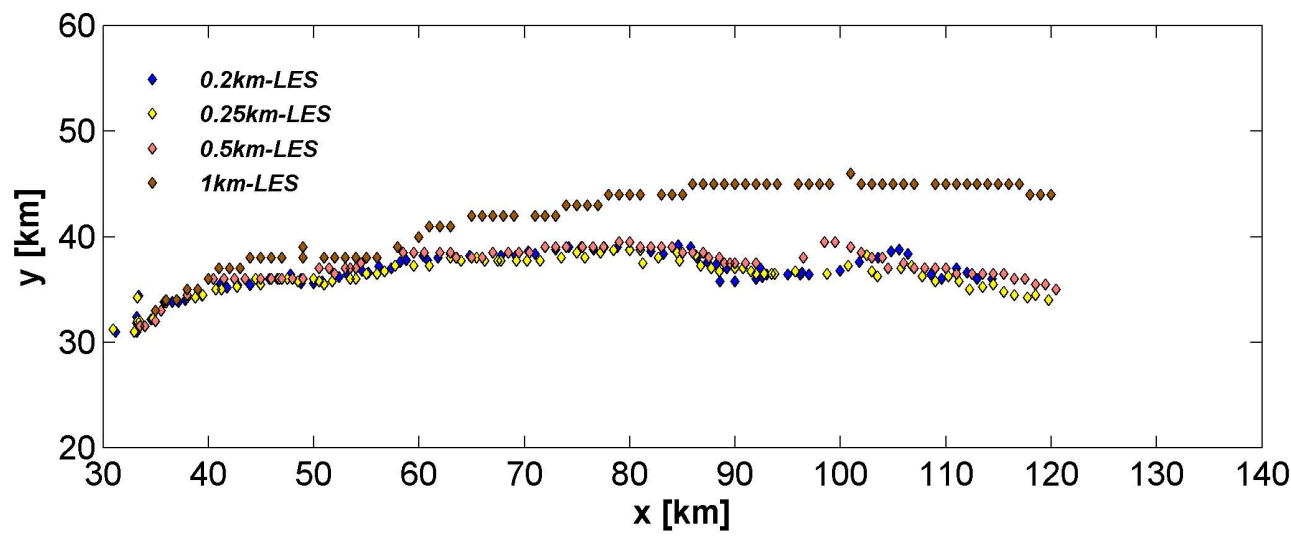

Fig. 5. As in Fig. 4 but using the LES turbulent closure.

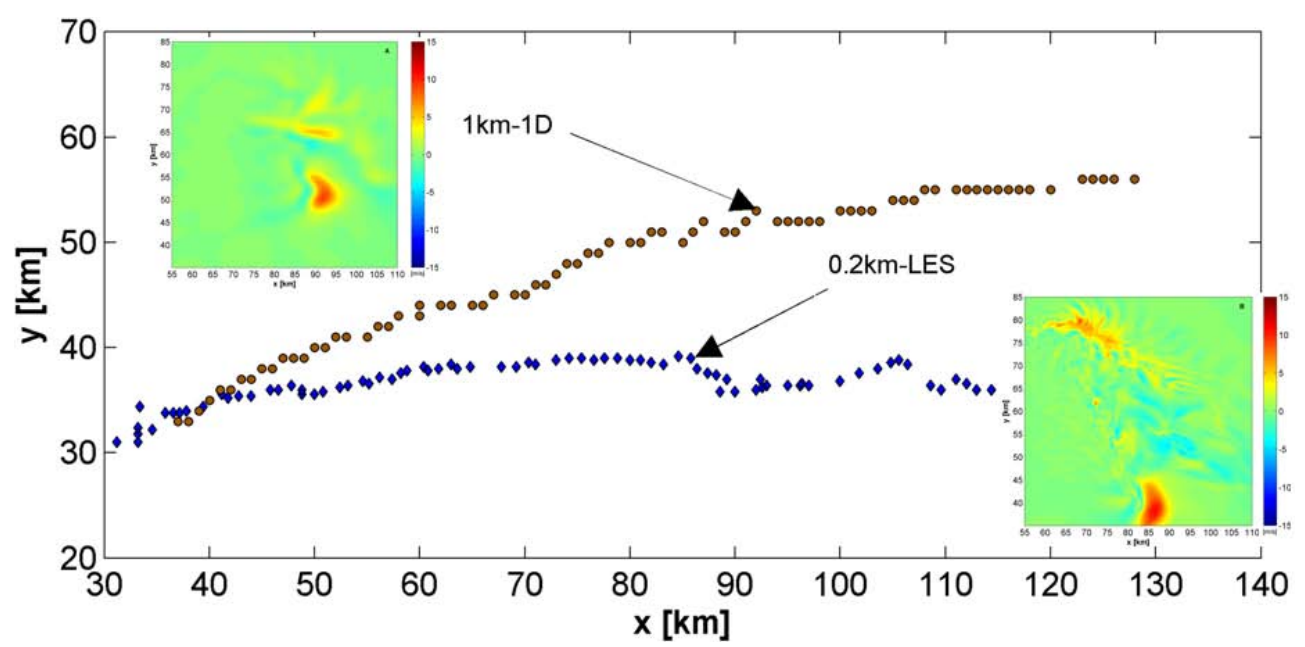

Fig. 6. Path of the core of the left right-moving cell in the first two hours of 1-km-1D (brown) and 0.2-km-LES (blue) run. In the left corner, horizontal cross-section of the vertical velocity $W$ for $1-\mathrm{km}-1 \mathrm{D}$ at $z=1.5 \mathrm{~km}$ a.g.1. after 90 min from initialization. In the right corner, horizontal cross-section of the vertical velocity $W$ for $0.2-\mathrm{km}$-LES at $z=1.5 \mathrm{~km}$ a.g.1. after 90 min from initialization. Arrows denote the positions along the paths. 

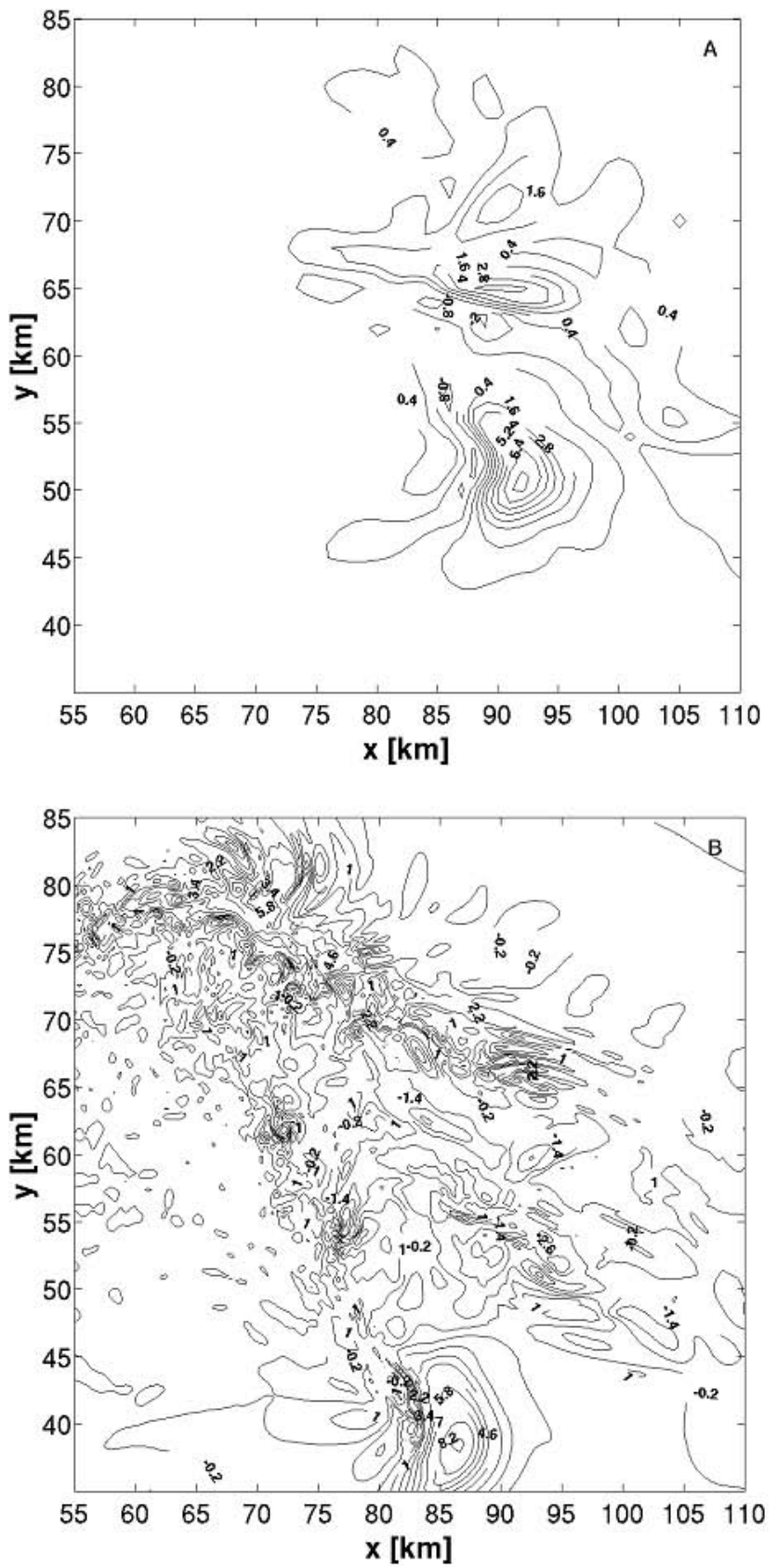

Fig. 7. Horizontal cross-section of the vertical velocity $W\left[\mathrm{~m} \mathrm{~s}^{-1}\right]$ at $1.5 \mathrm{~km}$ a.g.l. after $90 \mathrm{~min}$ from the initialization of the process. Contours at $1.2 \mathrm{~m} \mathrm{~s}^{-1}$. A 1-km-1D closure and $\mathbf{B}$ 0.2-km-LES closure.

for smaller ones. This kind of error could produce unpredicted floods. The difference of the convective patterns influences both intensity and spatial distribution of the rainfall. A map of the rainfall depth over the time window on which the $1 \mathrm{~km}-1 \mathrm{D}$ and $0.2 \mathrm{~km}$-LES paths are sensibly divergent has been produced in Fig. 8 . The time window covers $30 \mathrm{~min}$ between 1.5 and $2 \mathrm{~h}$ from the initialization of the process.

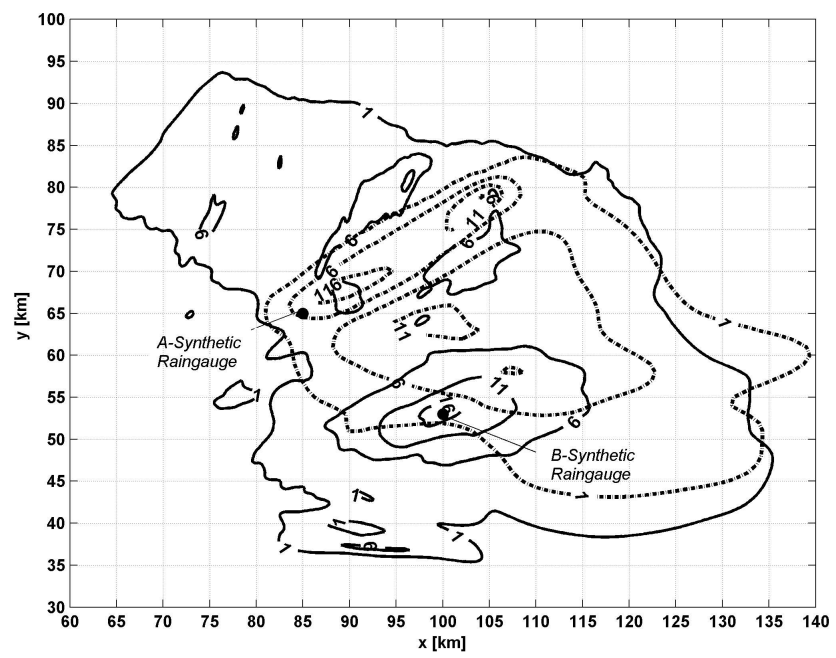

Fig. 8. Rainfall depth $h$ [mm], contoured at $5 \mathrm{~mm}$ interval, between $1.5 \mathrm{~h}$ and $2 \mathrm{~h}$ from the beginning of the convective scenario. Solid line: 0.2-km-LES run. Dash-dot line: $1 \mathrm{~km}-1 \mathrm{D}$ run. The position of synthetic raingauges A and B used in Fig. 9 are plotted.

The area covered by rainfall in the assumed truth-run $(0.2 \mathrm{~km}-\mathrm{LES})$ is higher than in the $1 \mathrm{~km}-1 \mathrm{D}$ run. This is a direct effect of the LES closure that makes the turbulent diffusion at fine resolution more efficient favouring the organization of smaller intense precipitating structures. In $1 \mathrm{~km}-1 \mathrm{D}$ run the most intense rainfall spots are displaced on the left flank of the gust front whilst the LES case concentrates high peaks in the area of the right-moving cell. The larger rainwater accumulation closer to the major cell in the truth-run has been confirmed by the intensification of the downdraft of the cyclonically rotating structures on fine resolutions.

\subsection{Hydrological interpretation of the uncertainty in a simplified atmospheric scenario}

Previous sections have shown how a fine resolution experiment, adopting LES turbulent closure, seems to converge in terms of kinematic properties of the convective flow field. The same correspondence has been found in terms of rainfall depth spatial pattern (not shown). Here, the existing differences between the two simulations discussed in Sect. 3.2 are evaluated from a hydrological point of view. To gain a clearer understanding of the spatio-temporal variability of the predicted rainfall depth, two synthetic raingauges (A and B) are supposed to be set, respectively, at the $(85 \mathrm{~km}, 65 \mathrm{~km})$ and $(100 \mathrm{~km}, 52 \mathrm{~km})$ location. They are visualized in Fig. 8 by two dots. In Fig. 9 the $1 \mathrm{~km}-1 \mathrm{D}$ predicted rainfall depth in both raingauges is compared with the assumed ground truth obtained by the $0.2 \mathrm{~km}$-LES simulation. For the synthetic raingauge $A$, the total rainfall depth is larger in the $1 \mathrm{~km}-1 \mathrm{D}$ run than the ground truth $0.2 \mathrm{~km}-\mathrm{LES}$ by a factor of $\sim 2$. Conversely, for the synthetic raingauge $B$ the total rainfall depth in the $1 \mathrm{~km}-1 \mathrm{D}$ is lower than the ground truth by a factor of $\sim 4$. Also the timing of the precipitation is different. 


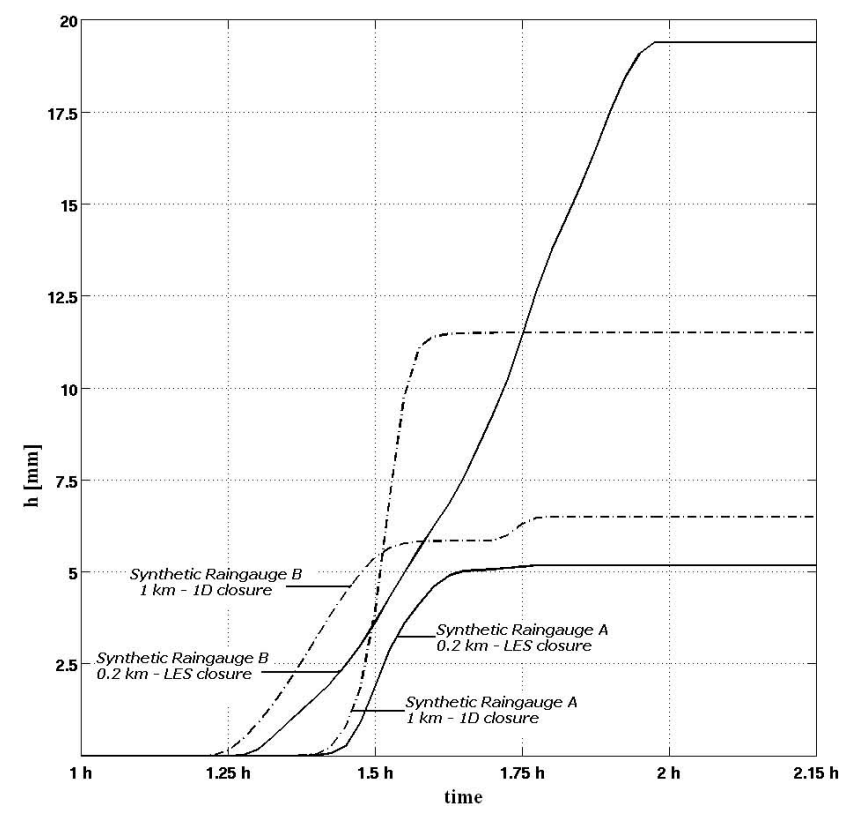

Fig. 9. Rainfall depth $h(t)[\mathrm{mm}]$ as observed by the synthetic raingauges $\mathrm{A}$ and $\mathrm{B}$.
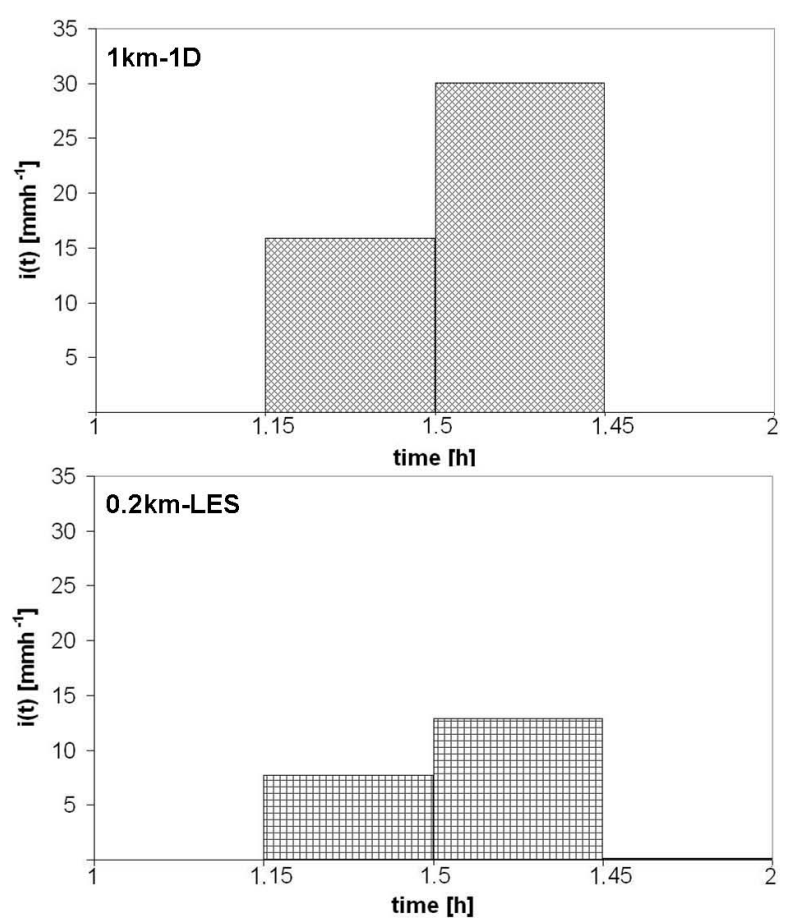

Fig. 10. Rainfall intensity $i(t)\left[\mathrm{mm} \mathrm{h}^{-1}\right]$ as observed by the synthetic raingauge $\mathrm{A}$ for the $1-\mathrm{km}-1 \mathrm{D}$ run (upper panel) and $0.2-\mathrm{km}$ LES (lower panel) run.
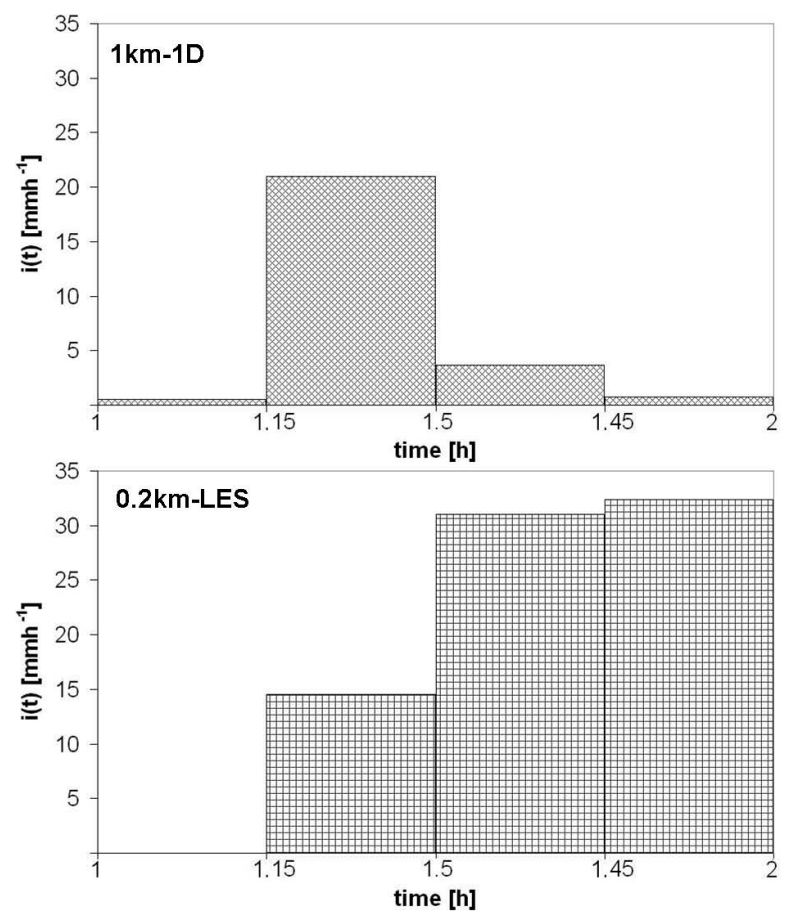

Fig. 11. The same as in Fig. 10 as observed by the synthetic raingauge $B$.

Another way to underline differences in the precipitating time series is through the use of the intensity $i(t)$ hyetograph. The hyetographs recorded for both simulations for the synthetic raingauge A are reported in Fig. 10 (1 km-1D on the left and $0.2 \mathrm{~km}$-LES on the right, respectively). Most of precipitation occurs over a very short timeslot of $30 \mathrm{~min}$ and this confirms the convective nature of the simulated scenario. Figure 11 shows the same for the raingauge $\mathrm{B}$ for $1 \mathrm{~km}-1 \mathrm{D}$ (upper panel) and $0.2 \mathrm{~km}-\mathrm{LES}$ (lower panel) runs, respectively. Here most of precipitation, for the $0.2 \mathrm{~km}-\mathrm{LES}$ case, occurs over a short timeslot of 45 min confirming, on one hand, the intermittent and convective nature of the simulated scenario and, on the other hand, the more intense rainfall activity of the right-moving cell at finer resolutions.

\section{Conclusions}

The capability of setting the model resolution at very fine scales does not a priori guarantee a reduction of uncertainty in numerical weather prediction in the so-called "terra incognita", i.e. in the horizontal resolution range of $1-0.1 \mathrm{~km}$. In particular, this work has proven how the difference in supercell propagation and the uncertainty in its physical representation are reduced when a suitable grid-spacing in combination with an adequate turbulent parameterization is chosen.

The use of the LES closure implies a faster and intense dynamics of the convective processes already at coarse resolution with respect to the 1D-closure. In LES-simulations the 
organizational mode of the convective overturning evolves from a classical supercell-like behaviour at coarser resolutions to a high precipitation supercell with multicellular convective organization on the left side of the gust front at $0.2 \mathrm{~km}$ grid-spacing.

In the $0.2 \mathrm{~km}$-LES run, the path of the right-moving cell increases the curvature with respect to the overall flow field structure. Convergence of the solutions is somehow shown when the resolution is increased to $\Delta=0.2 \mathrm{~km}$. This result is not confirmed by using $1 \mathrm{D}$-closure.

The difference of $20 \mathrm{~km}$ between the $1 \mathrm{~km}-1 \mathrm{D}$ simulated storm path and the one corresponding to the $0.2 \mathrm{~km}$-LES simulation produces, also in this highly idealized framework, remarkable differences in rainfall spatial distribution. It was shown that the rainfall depth is more concentrated and intense in the left-moving updrafts when 1D-closure is used at $\Delta=1 \mathrm{~km}$ whilst at finer resolutions using a LES closure, the precipitation occurs over a larger area and the highest amount of rainfall depth is mainly provided by the major cell.

In conclusion, the paper has shown that the turbulentclosure parameterization and resolution jointly influence the predictive ability of a model: a turbulent closure adequate to the resolution has to be chosen in order to achieve a proper modelling of deep moist convective processes.

Acknowledgements. This work was supported by the Italian Civil Protection Department. The authors are grateful to Richard Rotunno for the useful comments and discussions. The authors thank the referees for their careful revision that helped to improve the quality of this paper.

Edited by: S. Michaelides, K. Savvidou, and F. Tymvios Reviewed by: A. Papadopoulos and another anonymous referee

\section{References}

Adlerman, E. J. and Droegemeier, K. K.: The Sensitivity of Numerically Simulated Cyclic Mesocyclogenesis to Variations in Model Physical and Computational Parameters, Mon. Weather Rev., 130, 2671-2691, 2002.

Blackadar, A. K.: The Vertical Distribution of Wind and Turbulent Exchange in a Neutral Atmosphere, J. Geophys. Res., 67(8), 3095-3102, 1962.

Bougeault, P.: Physical parameterization for limited area models: some current problems and issues, Meteorol. Atmos. Phys., 63, 71-88, 1997.

Bryan, G. H., Wyngaard, J. C., and Fritsch, J. M.: Resolution Requirements for the Simulation of Deep Moist Convection, Mon. Weather Rev., 131, 2394-2416, 2003.

Buzzi, M.: Challenges in operational numerical weather prediction at high resolution in complex terrain, ETH, doi:10.3929/ethz-a005698833, 2008.

Davies, H. C.: Lateral boundary formulation for multi-level prediction models, Q. J. Roy. Meteor. Soc., 102, 405-418, 1976.
Doms, G., Forstner, J., Heise, E., Herzog, H.-J., Raschendorfer, M., Schrodin, R., Reinhardt, T., and Vogel, G.: A description of the Nonhydrostatic Regional Model LM. Part II: Physical Parameterization, Technical Report, 9-24, 2005.

Doswell III, C. A., Brooks, H. E., and Maddox, R. A.: Flash flood forecasting: An ingredients-based methodology, Weather Forecast., 11, 560-581, 1996.

Grabowski, W. W., Wu, X., Moncrieff, M. W., and Hall, W. D.: Cloud-resolving modeling of cloud systems during Phase III of GATE. Part II: Effects of resolution and the third spatial dimension, J. Atmos. Sci., 55, 3264-3282, 1998.

Kain, J. S., Weiss, S. J., Bright, D. R., Baldwin, M. E., Levit, J. J., Carbin, G. W., Schwartz C. S., Weisman, M. L., Droegemeier, K. K., Weber, D. B., and Thomas, K. W.: Some Practical Considerations Regarding Horizontal Resolution in the First Generation of Operational Convection-Allowing NWP, Weather Forecast., 23, 931-952, 2008.

Klemp, J. B. and Wilhelmson, R. B.: Simulations of right- and leftmoving storms produced through storm splitting, J. Atmos. Sci., 35, 1097-1110, 1978b.

Lilly, D. K.: The representation of small scale turbulence in numerical simulation experiments, in: Proc. IBM Scientific Computing Symposium on Environmental Sciences, IBM Form No. 3201951, 195-210, 1967.

Marshall, J. and Palmer, W. M.: The distribution of raindrops with size, J. Atmos. Sci., 5, 165-166, 1948.

Moller, A. R., Doswell III, C. A., and Przybylinski, R.: Highprecipitation supercells: A conceptual model and documentation. Preprints, 16th Conf. on Severe local Stormrs, Kananaskis Park, Alberta, Canada, American Meteorological Society, 52-57, 1990.

Moller, A. R., Foster, M. P., and Woodall, G. R.: The operational recognition of supercell thunderstorm environments and storm structures, Weather Forecast., 9, 327-347, 1994.

Paola, C., Foufoula-Georgiou, E., Dietrich, W. E., Hondzo, M., Mohrig, D., Parker, G., Power, M. E., Rodriguez-Iturbe, I., Voller, V., and Wilcock, P.: Toward a unified science of the Earth's surface: Opportunities for synthesis among hydrology, geomorphology, geochemistry, and ecology, Water Resour. Res., 42, W03S10, doi:10.1029/2005WR004336, 2006.

Petch, J. C., Brown, A. R., and Gray, M. E. B.: the impact of horizontal resolution on the simulations of convective development over land, Q. J. Roy. Meteor. Soc., 128, 2031-2044, 2002.

Roberts, N. M.: Assessing the spatial and temporal variation in the skill of precipitation forecasts from an NWP model, Meteorol. Appl., 15(1), 163-169, 2008.

Roberts, N. M. and Lean, H. W.: Scale-Selective Verification of Rainfall Accumulations from High-Resolution Forecasts of Convective Events, Mon. Weather Rev., 136, 78-97, 2008.

Rotunno, R. and Klemp, J. B.: On the rotation and propagation of simulated supercell thunderstorms, J. Atmos. Sci., 42, 271-292, 1985.

Rotunno, R. and Weisman, M. L.: A theory for strong, long-lived squall lines, J. Atmos. Sci., 45, 463-485, 1988.

Skamarock, W. C., Weisman, M. L., and Klemp, J. B.: Threedimensional evolution of simulated long-lived squall lines, J. Atmos. Sci., 51, 2563-2584, 1994.

Steppeler, J., Doms, G., Schättler, U., Bitzer, H. W., Gassmann, A., Damrath, U., and Gregoric, G.: Mesogamma scale forecasts 
using the nonhydrostatic model LM, Meteorol. Atmos. Phys., 82, 75-96, 2003.

Weisman, M. L. and Klemp, J. B.: The dependence of numerically simulated convective storms on vertical wind shear and buoyancy, Mon. Weather Rev., 110, 504-520, 1982.

Weisman, M. L. and Klemp, J. B.: The structure and classification of numerically simulated convective storms in directionally varying wind shears, Mon. Weather Rev., 112, 2479-2498, 1984.

Weisman, M. L. and Klemp, J. B.: Characteristics of isolated convective storms, in: Mesoscale Meteorology and Forecasting, edited by: Ray, P. S., American Meteorological Society, 331$358,1986$.
Weisman, M. L., Skamarock, W. C., and Klemp, J. B.: The Resolution Dependence of Explicitly Modeled Convective Systems, Mon. Weather Rev., 125, 527-548, 1997.

Wyngaard, J. C.: Toward Numerical Modeling in the "Terra Incognita”, J. Atmos. Sci., 61, 1816-1826, 2004.

Xue, M., Droegemeier, K. K., and Weber, D.: Numerical prediction of high-impact local weather: A driver for petascale computing. Petascale Computing: Al-gorithms and Applications, Taylor \& Francis Group, LLC, 103-124, 2007. 